\title{
Bi-temporal anodal tDCS during slow-wave sleep boosts episodic memory consolidation in high performers
}

\author{
Matthias Grieder,,$^{*}$ Yosuke Morishima, ${ }^{1}$ Stephanie Winkelbeiner, ${ }^{1,2}$ Sarah M Mueller, ${ }^{1}$ Kristoffer \\ Feher, ${ }^{1}$ Stefanie V Mueller, ${ }^{1}$ Thomas Dierks ${ }^{1}$ \\ ${ }^{1}$ Translational Research Center, University Hospital of Psychiatry, University of Bern, Bern, Switzerland \\ ${ }^{2}$ Center for Psychiatric Neuroscience, The Feinstein Institute for Medical Research, Manhasset, NY, USA
}

\begin{abstract}
Background: Sleep is crucial for sound memory functioning in humans. In particular, the slow waves that occur predominantly during slow wave sleep (SWS) are associated with hippocampus-dependent declarative memory consolidation. Making use of this association, boosting SWS to improve memory performance would be appealing for both healthy and memory-impaired populations. Transcranial direct current stimulation (tDCS) is a non-invasive brain stimulation that modulates the brain's excitability and has shown promising results in improving memory performance. However, owing to differing stimulation protocols and contradictory findings, there is insufficient evidence for the efficacy of tDCS-modulated hippocampal excitability on SWS and sleep-dependent memory consolidation.

Hypotheses: We aimed to enhance sleep-dependent memory consolidation and augment slow wave amplitudes.

Methods: We applied bi-temporal anodal tDCS to the left and right lateral temporal lobes of 31 healthy participants in a double-blind, sham-controlled, randomized crossover study. State-dependent tDCS was administered during slow wave sleep only. A pair-associate episodic memory task was used to assess sleep-dependent memory consolidation with face-occupation stimuli with baseline retrieval before sleep and delayed retrieval after sleep.

Results: Sleep-dependent memory consolidation was increased by tDCS only in participants who showed above-average performance (i.e. high performers) in baseline memory retrieval. Moreover, tDCS increased the slow wave amplitudes compared to sham.

Conclusions: When targeting a specialized brain mechanism such as memory consolidation with $t D C S$ during slow wave sleep, only those who were high performers at baseline achieved a memory boost.
\end{abstract}

Keywords: consolidation, episodic memory, slow wave sleep, tDCS, temporal lobe

\footnotetext{
${ }^{*}$ Correspondence to Matthias Grieder, PhD, Translational Research Center, University Hospital of Psychiatry, Bolligenstrasse 111, 3000 Bern 60, Switzerland. Tel.: +41 319328351; Fax: +41 319309961; E-mail: matthias.grieder@upd.unibe.ch.
} 


\section{Introduction}

Boosting memory abilities such as memorizing more or forgetting less is a preferable improvement for both memory-impaired as well as healthy populations. The most vulnerable memory system to disturbances is episodic memory, which stores and retrieves idiosyncratic experiences including spatiotemporal context [1]. Although episodic memory shares common properties with other declarative memory systems such as semantic memory (i.e. factual knowledge), it allows to mentally re-experience past events [2].

The framework of episodic memory with the most evidence assumes a two-stage memory system that encodes and stores episodic information [3]. In a first stage, initial encoding takes place, where information is temporarily stored in the hippocampus, while in a second stage memory representations are reactivated by the hippocampus during off-line states (e.g. sleep). This is thought to reflect consolidation by transforming and integrating the episodic information to long-term storage in the neocortex [4]. An alternative, more recent theory assumes that the hippocampus binds contextual information from surrounding brain areas. In contrast to the system consolidation theory, the contextual binding theory views consolidation as a supporting role of the hippocampus in modulating and maintaining associations in the neocortex [5]. Notwithstanding the divergences of the theories, the hippocampus is the main hub for forming and maintaining long-term episodic memory.

Thus, the consolidation stage entails the potential for augmentation of successful longterm storage of memories. Following from this, it is not surprising that memory consolidation and the effects of sleep are being investigated intensively [6, 7]. Particularly, non-rapid eye movement (NREM) sleep has been linked to hippocampus-based declarative memory consolidation [8]. NREM sleep can be divided into three stages, N1, N2, and N3 [9].
These stages differ in terms of sleep depth, which is commonly measured by means of an electroencephalogram (EEG) and subsequent frequency-band analysis. For example, stage $\mathrm{N} 3$ is characterized by slow waves (i.e. 0.5 to 2 $\mathrm{Hz}$ ) and is therefore referred to as deep sleep or slow wave sleep (SWS). Several studies showed that the particular properties of N3 are strongly correlated with declarative memory performance $[10,11]$. For example, Plihal and Born [12] found increased recall of word associations when the retention interval was set during early nocturnal sleep (i.e. with a high SWS ratio) as compared to REM sleep or the awake state. Similarly, Gais et al. [13] reported improved declarative memory performance in subjects who slept after learning as compared with subjects who did not sleep right after learning.

Consequently, enhancing memory consolidation during sleep could provide a solution for individuals with episodic memory deficits. Several attempts have been made to improve sleep-dependent memory consolidation with transcranial electric stimulation (TES). The initial studies reported promising results [14-16], but more recently, the findings were mixed [17-20].

One major challenge of assessing the efficacy of TES is due to the numerous stimulation protocols and varying parameters (e.g. transcranial alternating current stimulation [tACS] vs. transcranial direct current stimulation [tDCS], current density, electrode placement settings). For example, while Ladenbauer et al. [21] showed improved sleep-dependent memory consolidation after tACS in mild cognitive impairment, Lafon et al. [22] applied low-frequency tACS with implanted depth electrodes in patients with epilepsy. Despite the considerable advantage of direct contact between the implanted electrode and the target area, the stimulation did not lead to an entrainment of spindles or slow waves. Additionally, negative findings in healthy participants might have obscured an effect of individual baseline performance on 
responsiveness to TES. A number of studies have found that baseline performance of study participants might modulate the stimulation effect on the outcome performance [23-26]. Taken together, these examples suggest an efficacy-dependent brain-trait and brain-state variability of TES [27]. In addition, the interactions between stimulation protocols, task parameters, brain traits and states with TES responsiveness are yet to be determined.

In most studies that attempted a modulation of memory consolidation using TES, the left dorsolateral prefrontal cortex (DLPFC) was chosen as the target region for stimulation. The DLPFC, similar to the hippocampus, is strongly involved in episodic encoding and retrieval, especially when tested with verbal material [28-31]. Therefore, the main advantage of the DLPFC as opposed to the hippocampus as a stimulation site is its location on the surface of the cortex, which makes it more directly accessible for stimulation. However, a meta-analysis by Barham et al. [32] showed that TES over the DLPFC not only enhanced, but also disrupted declarative memory performance, or had no effect. Together, the available studies provide rich insights into the basic mechanisms of sleep-dependent memory consolidation as well as potentials and problems associated with TES as a tool for modulating memory performance.

One of the most intriguing questions in the declarative memory consolidation framework is whether sleep-dependent memory consolidation can be boosted by facilitating hippocampal activity during SWS. The hippocampus is a key brain region for transformation of newly encoded information and is involved in processes of reactivating encoded information in an orchestrated interplay with the neocortex [29]. Therefore, we applied anodal tDCS over the anterior temporal lobes to reinforce hippocampal reactivation during SWS, after the participants encoded paired associations. To homogenize the brain-state that is stimulated across subjects, we engaged tDCS only during SWS.
Our main hypothesis was that active tDCS improves sleep-dependent memory consolidation, the interaction between baseline and delayed stages of retrieving learned pair associations in particular. To account for potentially influencing effects of baseline performance on the delayed retrieval performance, we conducted an exploratory moderation analysis. In addition, we hypothesized that active tDCS enhances cortical SW amplitudes compared to sham.

\section{Methods}

\section{Participants}

Thirty-one right-handed healthy participants were recruited. All participants spoke German (or Swiss German) as their primary language, had normal or corrected to normal vision, and, for women, were in the luteal phase of their menstrual cycle [33]. In addition, participants were instructed to sleep less than 5 hours the night prior to the recording sessions. The rationale behind this was to provoke sufficient sleep pressure. Exclusion criteria were current or previous neurological or psychiatric disorders, severe somatic disorders, medical or neurological illness with possible influence on the brain physiology, intake of psychotropic medication or psychoactive substances, drug abuse or addiction including alcohol within the last two years, pregnancy (a pregnancy test was conducted on every female participant immediately before starting the recording procedure), current breast feeding, heart or head surgeries, tattoos close to the head, neck or shoulder and permanent make-up, as well as claustrophobia. Moreover, participants with a score of 7 or higher in the restless legs syndrome screening questionnaire (RLSSQ) or 10 or higher in the Epworth Sleepiness Scale (ESS) or 36 and higher in the Fatigue Severity Scale (FSS) were also excluded.

Data of 11 participants were discarded: seven did not show SWS in either of the two recording sessions, one individual scored higher than 7 on the restless legs syndrome 
screening questionnaire, two subjects had a score $\geq 36$ in the FSS, and one data set was discarded due to a technical failure. Thus, 20 participants ( 9 women) with a mean age of 24.0 years $(\mathrm{SD}=2.9 ; 21-32)$ were included.

All participants provided informed consent and the study was in accordance with the declaration of Helsinki. The Bern regional ethics committee approved the study (KEK-Nr. 083/14). Lastly, we had registered the study in the German registry of clinical trials (Deutsches Register Klinischer Studien, DRKS00009298).

\section{Episodic memory task}

An explicit episodic memory task was designed to assess sleep-dependent episodic memory consolidation. The stimulus material comprised 80 grey-scale male faces from the FERET database [34, 35]. Each face was associated with an occupation title (e.g. mason, Maurer in German). The within-subject design of the current study required two versions of the task (one for the active tDCS and one for the sham tDCS session), each of which contained 40 stimulus pairs. In the encoding phase, the participants were asked to learn the face-occupation associations that were displayed on a monitor. Face-occupation pairs were presented for five seconds, followed by a rating of how well the participant managed to picture the particular person executing the occupation. This step was introduced to facilitate the generation of episodic memory traces rather than mere semantic associations. Despite the fixed stimulus duration, the time to provide the rating was self-determined to improve memory consolidation at the individual (and group) level, instead of restricting learning speed. There were two subsequent encoding runs. Immediately after encoding, a baseline retrieval was performed, in which 20 of the 40 stimuli were retrieved by depicting only the face, but not the occupation. Participants were instructed to first rate the salary they associated with the face on a 4-step Likert-scale, and then name the associated occupation, which was registered by the experimenter. The salary rating served as an alternative dependent variable in case of floor effects in the free recall [36]. The delayed retrieval was performed 30 minutes after the participants woke up and was designed similarly to the baseline retrieval, with the remaining 20 faces of the total 40 stimuli. Stimuli presentation, time logging, and rating registration were performed using E-Prime (version 2.0, Psychology Software Tools Inc., Pittsburgh, PA, USA).

\section{Transcranial DC stimulation}

An Eldith DC-Stimulator (NeuroConn $\mathrm{GmbH}$, Germany) served as the stimulation device, and three rectangle-shaped rubber electrodes $(5 \times 7$ $\mathrm{cm})$, including two anodes (current split) and one cathode, were mounted underneath the EEG-cap onto the scalp. In the active tDCS condition, $2 \mathrm{~mA}$ direct current was administered for 120 s, preceded by a $5 \mathrm{~s}$ fade in and concluded by a $5 \mathrm{~s}$ fade out. A maximum of 15 stimulations were applied, resulting in a maximal stimulation duration of $30 \mathrm{~min}$ (in case a participant showed less than $30 \mathrm{~min}$ of SWS, fewer stimulations were applied). The sham $\mathrm{tDCS}$ was performed equally to the active condition, except that the 120 s stimulation period was replaced by four seconds of direct current at $2 \mathrm{~mA}$.

The rationale for the stimulation location is briefly outlined here. Due to the morphology of the temporal lobe, the hippocampus is not on the surface of the cortex and therefore cannot be targeted directly. Hence, the target region had to be functionally connected with the hippocampus as well as located on the surface of the cortex. According to the review by Svoboda et al. [37], the brain regions fulfilling these criteria for autobiographical memory processes are the dorsomedial prefrontal cortex, the inferior parietal lobe, and the lateral temporal cortex. Note that the functional connectivity reported in Svoboda et al. [37] was based on awake resting-state data. Moreover, Andrade et al. [38] did not find any suprathreshold regions 
that showed greater functional connectivity between the hippocampus and any cortical regions during SWS as compared to $\mathrm{N} 2$ or the awake state.

Additionally, we performed a functional connectivity analysis to determine the target location. For this purpose, we used the restingstate fMRI data on an independent sample $(\mathrm{N}=$ 100) from the Human Connectome Project [39]. The dataset was composed of resting state fMRI echo-planar images (1200 volumes; repetition time $=720 \mathrm{~ms}$; echo time $=33.1 \mathrm{~ms}$; field of view $=208 \times 180 \mathrm{~mm}$, plane resolution $=$ $2 \times 2 \mathrm{~mm}$; slice thickness $=2 \mathrm{~mm}$; multiband factor $=8$; phase encoding direction $=\mathrm{R} / \mathrm{L}$ ) and artifacts were extensively removed and normalized to the Montreal Neurological Institute (MNI) space [40]. We additionally applied spatial smoothing with a $6 \mathrm{~mm}$ fullwidth half-maximum Gaussian kernel.

Next, we generated a bilateral hippocampus mask (Figure S1A) from the probabilistic atlas included in the SPM Anatomy toolbox [http://www.fzjuelich.de/inm/inm-

1/DE/Forschung/_docs/SPMAnatomyToolbox/ SPMAnatomyToolbox_node.html, 41]. In addition, we created mask images of gray matter (GM), white matter (WM), and cerebrospinal fluid (CSF) voxels in the MNI space to regress out the global fluctuation of blood oxygen level-dependent (BOLD) signals. We constructed 15 confounding regressors: 13) time series of GM, WM, and CSF, 4-9) six head motion parameters, 10-15) first order derivative of the six head motion parameters. After extracting BOLD time series of the hippocampus, as well as the whole brain BOLD signals, we regressed out the 15 confounding regressors and calculated voxel-wise correlations between the hippocampal time series and whole brain. We converted Pearson's correlation coefficients to z-scores using Fisher transformation. These transformed values were entered into a one sample t-test for a random effect analysis.
The results yielded several voxel-clusters that exceeded the voxel-wise family-wise error (FWE)-corrected threshold $\left(\mathrm{T}_{(1,99)}=5.22, \mathrm{p}\right.$ $<.05)$. Figure $\mathrm{S} 1 \mathrm{~B}$ illustrates the brain regions at the surface of the cortex that are functionally connected with the hippocampi. In addition to the postcentral gyrus, lateral occipital cortex, and middle to superior frontal gyrus, the cluster ranging from anterior to posterior middle and superior temporal lobe were particularly evident (for a complete list of significant clusters, see Table S1). Taken together, the bilateral lateral temporal lobes appeared to be the best suited target areas for our stimulation approach. Therefore, the position for the two $5 \times 7 \mathrm{~cm}$ tDCS electrodes was over EEG 10-20 electrode T7 for the left and T8 for the right hemisphere. The tDCS reference electrode was placed on the neck.

After the target area was established, the direction of the current flow was determined. With the aim of reinforcing hippocampal activation, anodal stimulation was chosen due to its facilitating effect [42-45]. An additional cathodal stimulation would have added the possibility of interpreting the directionality of a possible tDCS effect. However, a metaanalysis [46] suggested anodal stimulation for effects on cognition, given that cathodal stimulation sometimes induces inhibition in motor regions. Therefore, we refrained from additionally testing the cathodal stimulation condition in our study. The exact stimulation parameters can be found in Figure 1.

As outlined in the introduction, tDCS was engaged during SWS as monitored visually by the experimenters using online EEG inspection. This approach entails a more homogeneous brain state that is stimulated across subjects and might account better for inter- and intra-subject variability, as for example offline or sleep stage-independent tDCS application. Moreover, putative tDCS effects on memory consolidation could be attributed to the SWS stimulation, rather than to unidentified sleep stages. Finally, a current flow simulation was conducted in order to 
verify that this novel tDCS setup would generate increased current flow in the hippocampi (HD-Explore, Soterix Medical Inc., New York, NY, USA). As can be seen in Figure S2, there was sufficient indication to verify the increased current flow.

\section{Polysomnography}

Twenty-two sintered silver chloride ring electrodes were mounted on a cap in a 10-20 fashion and then connected to a 16-bit BrainAmp Standard amplifier (Brain Products $\mathrm{GmbH}$, Gilching, Germany). Impedances were maintained below $10 \mathrm{k} \Omega$, the sampling rate was $1 \mathrm{kHz}$, the input range $3.28 \mathrm{mV}$, and an online band-pass filter was applied ranging from 0.1$1000 \mathrm{~Hz}$. The reference electrode was placed at $\mathrm{Cz}$, and the ground electrode at POz. During the sleep recording, an online notch-filter at 50 $\mathrm{Hz}$ was applied to suppress environmental artifacts. In addition to the EEG montage, adhesive electrodes were placed underneath each eye for the electrooculogram, under the chin for the electromyogram, and on the shoulder and chest, respectively, for the electrocardiogram. For these recordings, a bipolar ExG BrainAmp 16 was used (Brain Products $\mathrm{GmbH}$, Gilching, Germany). The amplifier's specifications were equal to those of the BrainAmp Standard, except that the sampling rate was $5 \mathrm{kHz}$.

\section{Experimental procedure}

Prior to the experimental recordings, all participants completed several questionnaires (ESS, FSS, Pittsburgh sleep quality index form, RLSSQ), which were evaluated before the first recording session. Moreover, participants were informed about the study procedure and made aware that on one recording session they receive active tDCS and on the other sham. On the evening of the first recording session, participants completed the ESS again, followed by the pregnancy test for female participants. We applied the locally anaesthetizing Emla Crème 5\% (AstraZeneca,
London, UK) to the scalp where the tDCS electrodes would be mounted, as pilot recordings showed that participants awoke with the engagement of the tDCS. Moreover, Guleyupoglu et al. [47] demonstrated that abolishing skin sensation improves blinding of the participants. Next, the participants were introduced to the self-guided episodic memory paradigm. After the learning phase and immediate baseline retrieval, the EEG cap was mounted to the participant's head including the tDCS electrodes. The first recording was a 6 min $40 \mathrm{~s}$ awake resting EEG in a seated position, with alternating eyes closed and eyes open conditions. Next, the participant lay down on the stretcher that belonged to an MRsimulator device (MRI Simulator System, Model No. PST-100355, Psychology Software Tools, Inc., Sharpsburgh, USA). This way we tested whether participants can sleep in a noisy and space-constrained MRI-environment. To conclude the setup, a mock head coil was attached, and the EEG impedances were double checked. Before the main sleep recording, we conducted another resting EEG measurement in supine position. After that, the participants were informed that the sleep recording was to be started and that they could relax and sleep. During the sleep recording, the simulated MR acoustic noise was engaged using the SimFxTM Software (Psychology Software Tools Inc., Pittsburgh, PA, USA). Ideally, after the participants slept for a whole sleep cycle or a maximum of two hours, they were woken and released from the MRsimulator. We chose a two-hour sleep design for two reasons: first, for the planned subsequent sleep study in an MRI, a wholenight sleep design is not feasible; second, previous studies have shown declarative memory consolidation benefits from daytime naps $[48,49]$. To assess blinding of the participants, we assessed the subjective impression of whether or not the true stimulation had been applied. After 30 minutes of waking up time, the consolidated delay retrieval was performed. A schematic overview 
of the experimental procedure is shown in

Figure 1.

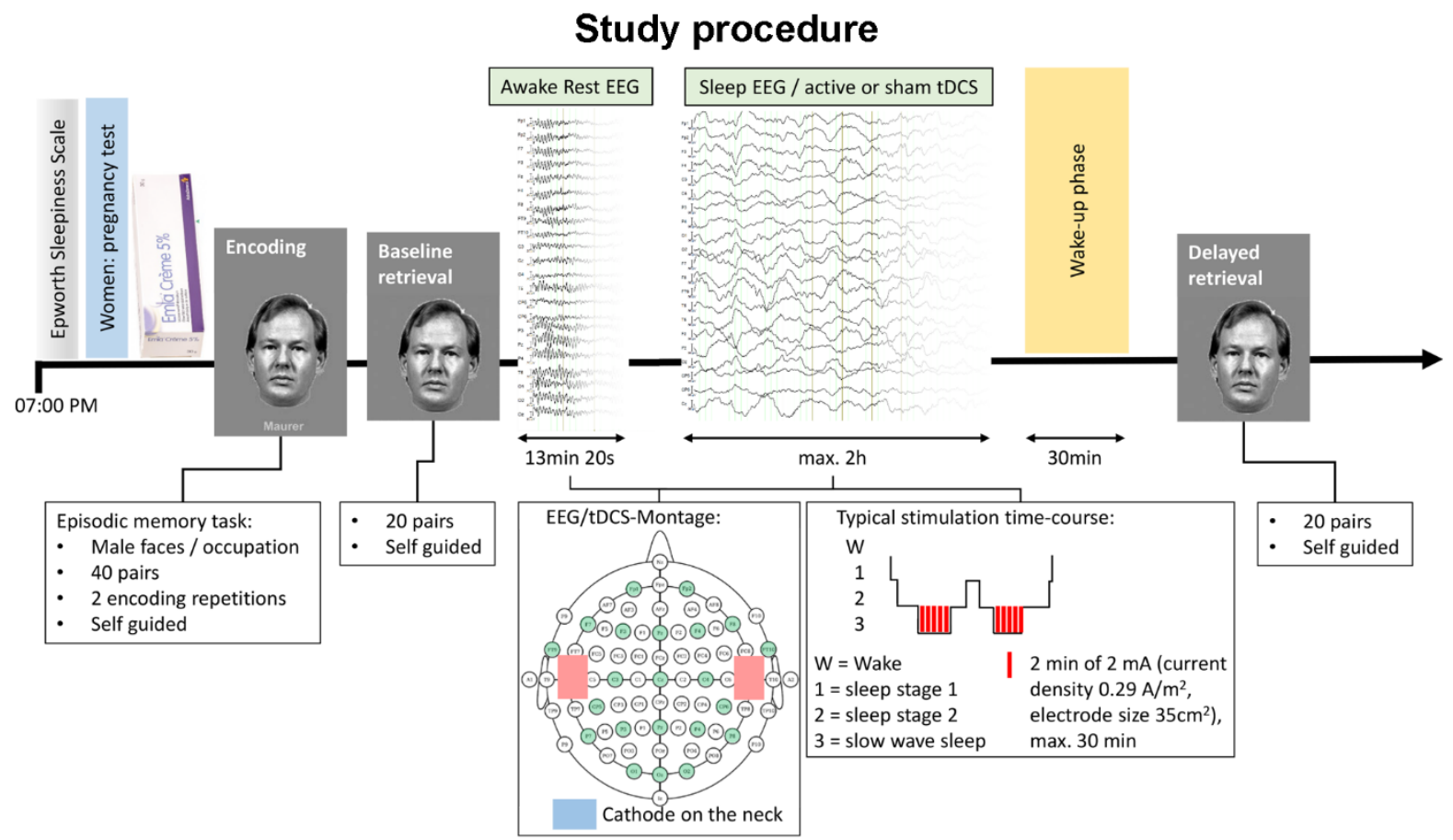

Figure 1: Participants underwent this study procedure twice with at least 4 weeks in between sessions, once with active tDCS and once with sham-stimulation. Red rectangles represent anodal tDCSelectrodes; the blue rectangle represents cathodal tDCS-electrode. Green shaded circles represent the 22 recording EEG channels overlaid on the 10-20 international electrode system.

\section{Data processing and analysis}

\section{Sleep staging}

The sleep staging was performed offline by MG and SW independently and in accordance with the AASM Manual [9]. As recommended, the sleep-EEG was low-pass filtered at $0.5 \mathrm{~Hz}$, high-pass filtered at $35 \mathrm{~Hz}$, and a Notch at 50 $\mathrm{Hz}$ was applied. Next, the EEG was segmented into 30-s epochs, and each epoch was staged into either wakefulness (W), NREM 1 (Ni), NREM 2 (N2), NREM 3 (SWS), or REM (R). Additionally, epochs contaminated by tDCS ramp-up or ramp-down artifacts were labeled as NX.

\section{EEG preprocessing}

EEG channels that showed a poor signal throughout the recording were discarded. To systematically exclude data contaminated by tDCS artifacts, an algorithm was applied to mark such data parts. In particular, EEG data during ramp-up and ramp-down of the tDCS caused channels to saturate for a certain period of time. Such saturated data was identified by checking for maximal and minimal amplitudes of $+/-3200 \mu \mathrm{V}$. Next, a high pass filter of 0.5 $\mathrm{Hz}$, a low pass filter of $35 \mathrm{~Hz}$, and a notch filter at $50 \mathrm{~Hz}$ were applied, followed by computing the average reference. Contralateral referencing against auricular or mastoid electrodes was not advised due to the proximity of the tDCS electrodes. Remaining bad channels were interpolated. A manual visual inspection was also performed, and bad intervals were marked. Then, all clean data was segmented starting from the first stimulation and finishing at the end of the recording. From this segmented data, data that was rated as $\mathrm{N} 2$ or SWS were segmented. The last segmentation involved the extraction of equally long epochs of $10 \mathrm{~s}$ that were subjected to further analysis.

Slow wave detection 
To quantify SWS, we applied automatic SW detection of the sleep EEG data. According to Iber [9], SWs can be defined as oscillations with a frequency range between 0.5 and $2 \mathrm{~Hz}$, and a peak-to-peak amplitude that exceeds $75 \mu \mathrm{V}$. To detect the periods fulfilling these criteria, we applied a band-pass filter with $0.5-2 \mathrm{~Hz}$. Then, for each channel, we detected troughs and crests of EEG signals using the "findpeaks" MATLAB function (Version 8.5.0.197613; The MathWorks Inc., Natick, MA, USA), and calculated the peak-to-peak amplitude of each oscillating cycle. More precisely, each peak-topeak amplitude of an oscillatory cycle that exceeded the threshold of $75 \mu \mathrm{V}$ was recognized as a slow wave. Our MATLAB script saved the amplitude of every identified slow wave.

\section{tDCS effects}

For the unlikely but possible case that variables of no interest such as learning duration, SWS time, and sleepiness were significantly different between and active and sham tDCS, we employed non-parametric Wilcoxon tests to rule out such undesired effects on our main study outcome.

We tested our main hypothesis, namely improved sleep-dependent memory consolidation in the active tDCS compared to sham, with a moderation analysis [23]. We used the SPSS PROCESS macro (version 3.0 [50]), to test the moderating effect of baseline retrieval on the tDCS effect as assessed by the delayed retrieval performance. We applied the software's model 1 with tDCS (active or sham) as the independent variable, delayed retrieval performance as the dependent variable, and baseline retrieval performance as the moderator variable. The Johnson-Neyman method was conducted to thoroughly inspect the tDCS effect as a function of baseline retrieval performance $[50,51]$. Finally, baseline retrieval performances were grouped by centering the mean, $-S D$, and $+S D$, which provided an illustrative interpretation of the tDCS $x$ baseline retrieval performance interaction.

In addition to the moderation analysis, we performed a $2 \times 2$ repeated-measures analysis of variance (ANOVA) to test the effects of retrieval delay (baseline/ delayed) and tDCS condition (active/ sham) on the group level. The second hypothesis was assessed by comparing the SW amplitude (SWamp) of active and sham tDCS using non-parametric Wilcoxon tests.

\section{Results}

Of the 20 participants, only one participant reported nausea shortly after waking up. No other adverse effects in relation to the experimental procedure were reported. Moreover, the blinding of the participants to the tDCS condition was successful (Table S2). On average, $19.3 \%$ EEG data in the active and $15.3 \%$ in the sham tDCS conditions was discarded due to artifacts, mainly caused by tDCS fade in and out. There was no significant difference of discarded EEG data between tDCS conditions (Wilcoxon $Z=-1.53, p=0.13$ ).

\section{Sleep stages}

The interrater reliability of sleep staging was high (Cronbach's $\alpha=0.86$; Cohen's Kappa: $x=$ $0.738, \mathrm{SE}=0.006, p<.001$ ). REM sleep was not present in the sleep data, which can be expected in studies conducted in noisy environments and with a focus on the first sleep cycle only [52]. Total sleep time (TST) as well as sleep stage duration data can be viewed in Table 1. No statistically significant differences between sham tDCS and active tDCS were found. 
Table 1: Descriptive statistics of sleep stage architecture, memory retrieval scores, and SW characteristics for the tDCS conditions (mean $\pm \mathrm{SD}$ ).

\begin{tabular}{lllll}
\hline & sham tDCS & active tDCS & Z & $p$ \\
\hline TST & $70.6 \pm 25.2$ & $69.1 \pm 25.5$ & 0.07 & 0.94 \\
W & $23.4 \pm 19.4$ & $27.7 \pm 24.1$ & 0.54 & 0.59 \\
N1 & $15.3 \pm 10.0$ & $17.4 \pm 11.3$ & 0.75 & 0.46 \\
N2 & $26.5 \pm 19.7$ & $27.3 \pm 18.6$ & 0.34 & 0.74 \\
SWS & $25.6 \pm 16.6$ & $22.7 \pm 22.4$ & 0.95 & 0.34 \\
NX (artifact) & $3.3 \pm 2.4$ & $1.8 \pm 2.9$ & 1.68 & 0.09 \\
Baseline retrieval & $11.6 \pm 4.9$ & $12.4 \pm 4.2$ & N/A & \\
Delayed retrieval & $10.0 \pm 3.2$ & $11.1 \pm 5.0$ & N/A & \\
SWamp & $91.0 \pm 6.4$ & $93.1 \pm 5.4$ & -2.1 & $0.03^{*}$ \\
Nr of SW & $725.5 \pm 711.3$ & $676.3 \pm 910.3$ & -0.41 & 0.68 \\
\hline
\end{tabular}

TST, total sleep time; W, wake; N1, NREM sleep stage 1; N2, NREM sleep stage 2; N3 (SWS), NREM sleep stage 3 (slow wave sleep); SWamp, slow wave amplitude; SW, slow waves; ${ }^{*}, p<.05$.

\section{Episodic memory consolidation}

The ANOVA showed a significant main effect of retrieval delay $\left(\mathrm{F}_{(1,19)}=14.49, p=.001\right)$, but not of tDCS condition $\left(\mathrm{F}_{(1,19)}=0.86, p=.37\right)$. Expectedly, no retrieval delay-tDCS condition interaction was found $\left(\mathrm{F}_{(1,19)}=0.09, p=.77\right.$; for mean retrieval scores and SD, see Table 1 ). Thus, retrieval was lower in the delayed condition than at baseline, with no tDCS effect on memory retrieval at the group level. Figure 2 shows individual memory performance and emphasizes that in the sham tDCS condition a higher baseline performance was associated with lower consolidated memory performance. In contrast, subjects with a lower baseline performance retrieved more items in the consolidated recall condition. This pattern was altered in the active tDCS condition, yielding more horizontally or positive slopes for higher baselines and less negative slopes for lower baselines.

Wilcoxon tests suggested that neither effects of learning duration, time spent in SWS, nor sleepiness influenced the main outcome of this study (learning duration: $\mathrm{Z}=$ 1.27, $p=.20$; SWS duration: $Z=-0.95, p=.34$; ESS: $\mathrm{Z}=-0.23, p=.82$ ).
Moderation analysis showed that the overall model was significant $\left(\mathrm{F}_{(3,36)}=28.64, \mathrm{R}^{2}\right.$ $=0.70, p<.0001, \mathrm{f}^{2}=2.33$, power $\left.=0.99\right)$. The two single effects of tDCS ( $\mathrm{T}=-2.53, p=.016$ ) and baseline memory performance $(\mathrm{T}=4.65, p$ $<.0001$ ) as well as their interaction were significant $(\mathrm{T}=2.94, p=.0057)$, which increased the explained variance of the model $\left(F_{(1,36)}=8.64, R_{\text {change }}^{2}=0.071\right)$. This suggested a tDCS effect on memory consolidation.

The Johnson-Neyman method revealed that only participants who remembered 15 associations or more benefited from the tDCS, whereas $\mathrm{tDCS}$ would even worsen performance of those with a baseline retrieval of 6 or less (Figure 3A, Table S3). In line with this result, the episodic memory performance centering ($\mathrm{SD}$, mean, +SD) showed that only the high performers' sleep-dependent memory consolidation was positively influenced by active $\mathrm{tDCS}$ (Figure $3 \mathrm{~B} ; \mathrm{M} \Delta=2.74, \mathrm{SE}=1.07, \mathrm{t}=$ 2.57, $\mathrm{p}=.01$ ). In contrast, no significant tDCS effect was elicited in the average $(\mathrm{M} \Delta=0.48, \mathrm{SE}$ $=0.75, \mathrm{t}=0.65, \mathrm{p}=.52)$ and low-performing participants $(\mathrm{M} \Delta=-1.77, \mathrm{SE}=1.08, \mathrm{t}=-1.64, \mathrm{p}=$ $.11)$. 
A

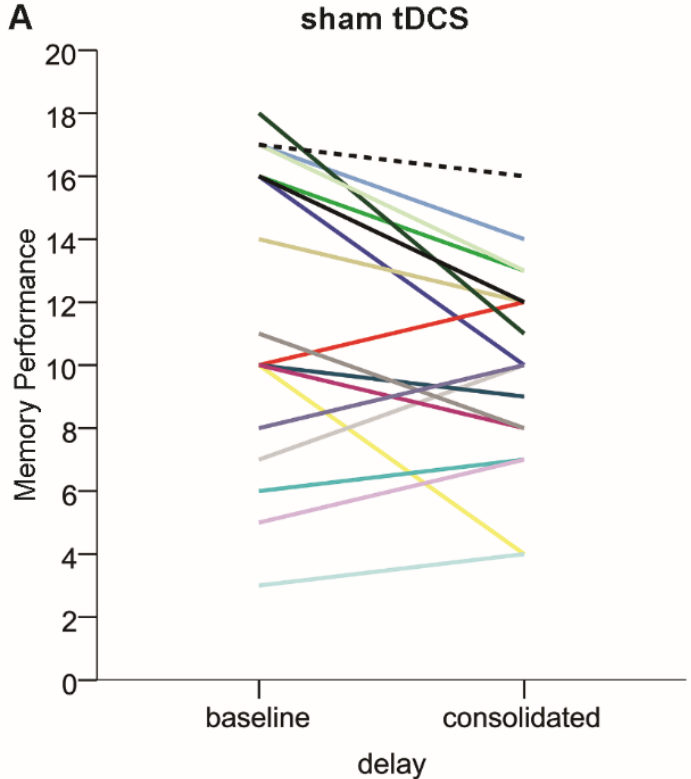

B

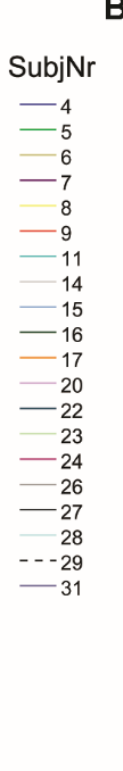

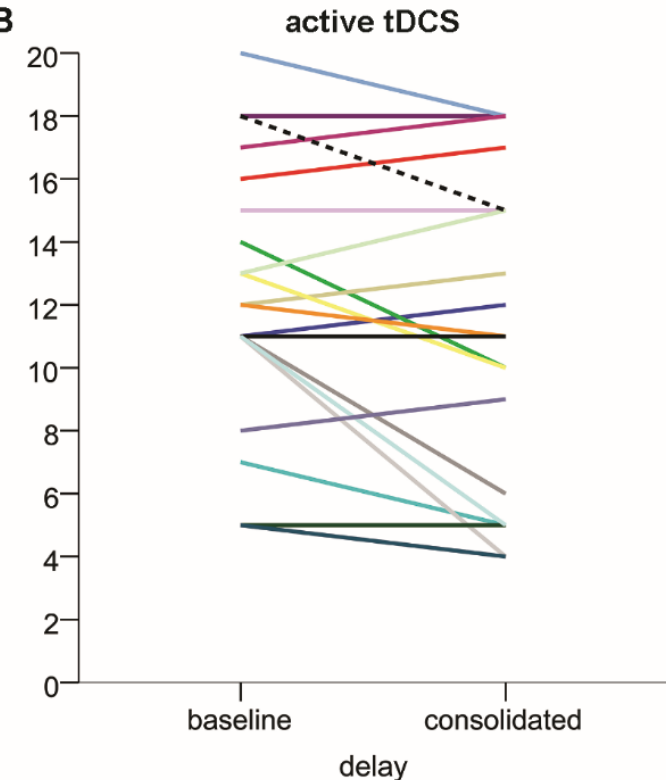

SubjNr

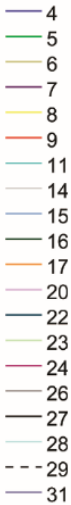

Figure 2. Individual memory retrieval performances at baseline and delayed. (A) shows scores for sham tDCS, (B) for active tDCS.

A

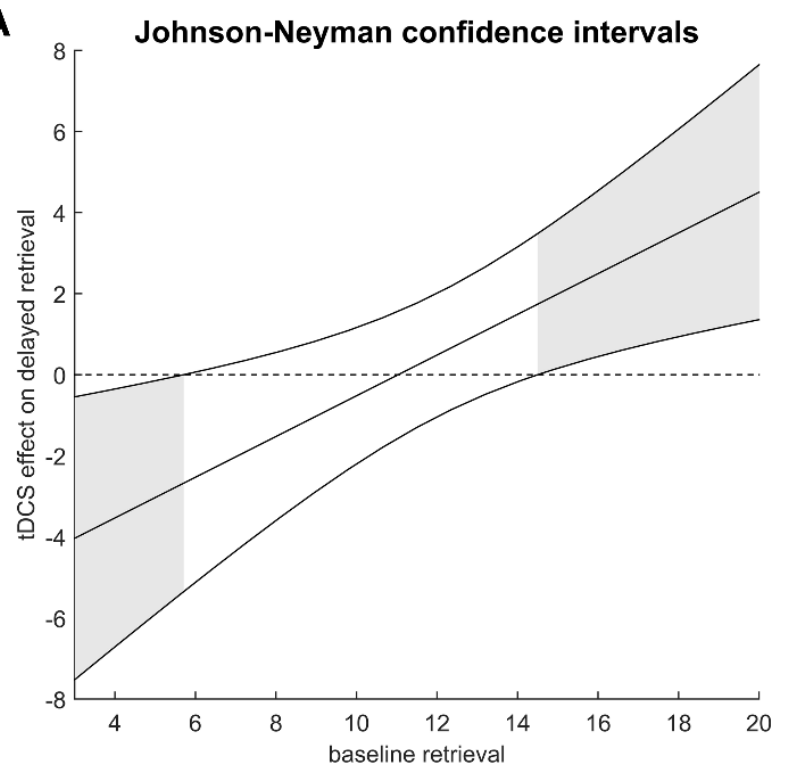

B Episodic memory performance centering

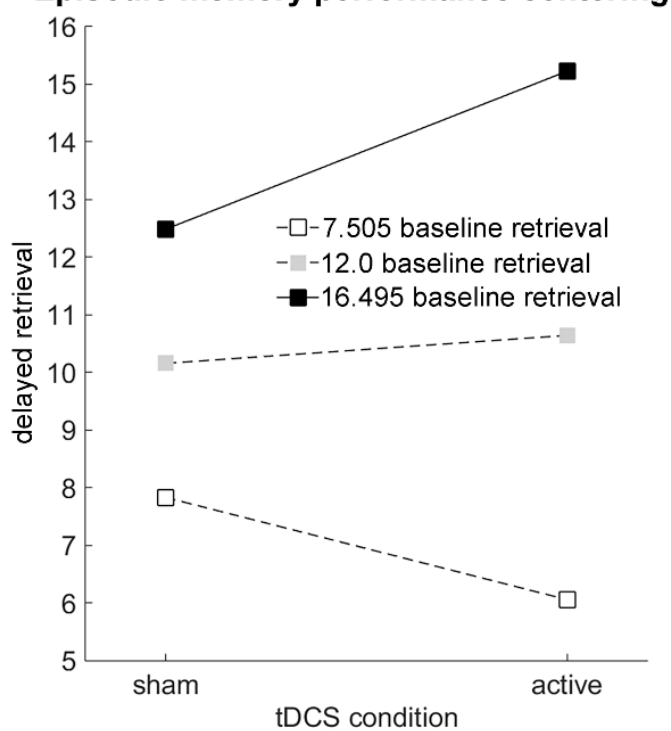

Figure 3. (A) Baseline retrieval predicts beneficial or deteriorating tDCS effect on the delayed memory retrieval in high $(\geq 15)$ and low $(<6)$ performers. Grey shaded areas between $95 \%$ confidence intervals represent significant tDCS effects. (B) Moderator effect of baseline retrieval on delayed retrieval as a function of tDCS condition. The three groups represent performance centering at -SD (white squares), mean (grey squares), and +SD (black squares).

\section{Slow wave amplitudes}

The slow wave amplitude was significantly increased in the active tDCS condition compared to sham as assessed with the Wilcoxon test $(\mathrm{Z}=-2.1, p=.03$, Table 1, Figure $4 \mathrm{~A})$. Note that this finding applied only to the analysis including all SWamp after the first stimulation until the end of the recording, but not to SWamp that occurred only during stimulation. The number of slow oscillations that exceeded the $75 \mathrm{mV}$ threshold and that were subsequently subjected to the SWamp analysis did not differ between the tDCS conditions (Table 1). Figure 4B illustrates that the individual effect of active tDCS on SWamp varied, with a majority of increased amplitudes in the active tDCS condition. 
A

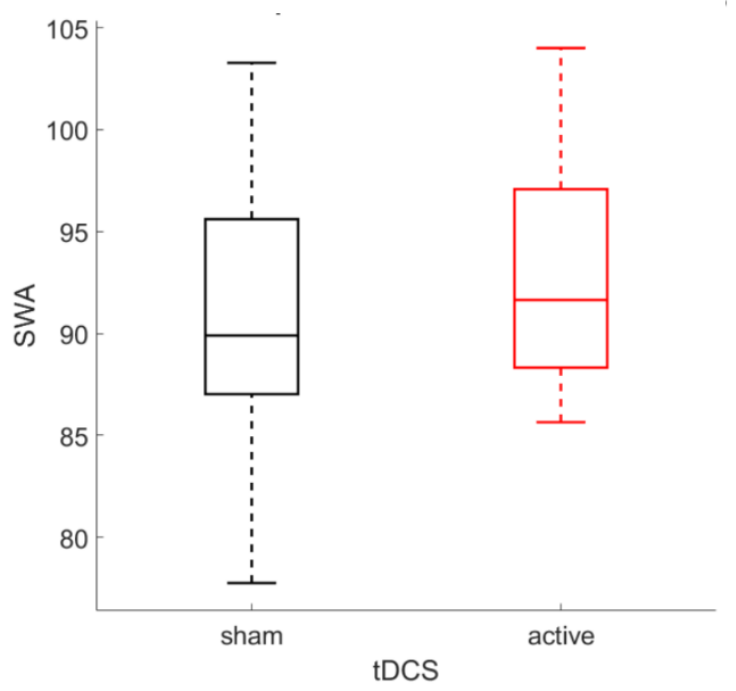

Figure 4. (A) Boxplot illustrates group effect. (SWamp) for the tDCS conditions.

\section{Discussion}

In this study, we attempted to boost sleepdependent episodic memory consolidation, and this boost was accomplished only in participants with high baseline memory performance. Participants who performed on an average level or below did not benefit from tDCS. Therefore, it was not surprising that the repeated-measures ANOVA did not yield any stimulation effect, since the majority of the sample performed on an average level. For this purpose, we evaluated the outcome of the exploratory moderation analysis in more detail. The moderation model explained a substantial amount of the $70 \%$ of the explained variance. In the study of Habich et al. [23], just $50 \%$ of the variance was explained. Moreover, the tDCS effect on consolidation increased the explained variance by $6 \%$; the $7 \%$ in our study is comparable.

In contrast to previous studies that found stronger tDCS effects on low performers [23-25, 53], the high performers in our study showed the anticipated tDCS effect. It is important to note that none of the studies applied comparable tasks, stimulation timing, or stimulation sites. An exception is Habich et al. [23], who conducted an episodic memory
$B$

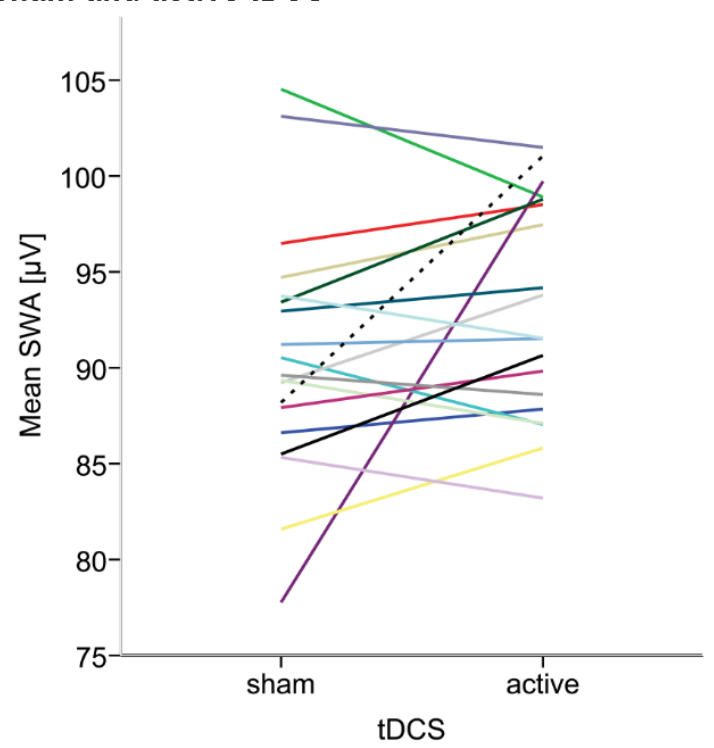

task and introduced delayed retrieval intervals. The major difference from our study was that stimulation was administered during encoding in the wake state, as was the consolidation period. The results indicate that in healthy participants, the potential for memory consolidation improvement by means of tDCS depends on the baseline performance and on the state of wakefulness or sleep, respectively.

One might argue that the available evidence favors tACS (or oscillatory tDCS) over tDCS as a capable tool for enhancing SWs and declarative memory performance during sleep (see meta-analysis [32]). Yet, the efficacy of both stimulation types is inconclusive across these studies. Moreover, most of the experimental setups of studies using tDCS or tACS entail frontal lobe stimulation. In the framework of system consolidation, it appears plausible to entrain SWs that predominantly occur frontally within the neocortex [54]. However, according to the postulated mechanism, the consolidation loop is initiated by the hippocampus, which does not necessarily produce SWs that are commonly measured at the scalp surface [6]. Therefore, anodal tDCS was our stimulation type of choice 
to facilitate hippocampal activity during SWS and to increase SWamp via the pathway between subcortical structures (i.e. hippocampus, thalamus) and the neocortex. Hence, in comparison with the previous studies, we attempted to bridge the gap of knowledge on the ability and efficacy of enhancing sleep-dependent declarative memory consolidation by applying TES to temporal (vs. frontal) brain regions in a brainstate dependent manner.

The secondary outcome, the tDCS effect on SWamp, was confirmed as well, albeit with an interesting side effect. In particular, merely contrasting SWamp during stimulation between active and sham tDCS yielded no significant effect. When all SWamps after the first stimulation were included in the analysis, however, the contrast was significant, with active tDCS inducing elevated SWamp. We offer two explanations: First, taking all SWamps after the first stimulation simply increased the statistical power by augmenting the number of SWamps for comparison. Second, the version that comprised only SWamps during stimulation was unable to capture after-effects to the same extent as the version that included all SWamps. In other words, the facilitating effect of anodal stimulation might have been stronger shortly after the stimulation than during the administration.

Some limitations merit comment. First, the study design included only anodal stimulation, with no cathodal stimulation, which would theoretically worsen sleepdependent memory consolidation. Thus, a mechanistic effect of anodal stimulation cannot be determined. However, as outlined in the introduction, the dual polarity effect more frequently found in motor studies does not seem to apply to the cognitive (declarative) memory domain. Bruckner and Kammer [55] found semantic improvements in both anodal and cathodal stimulation conditions. Second, the intended increase in hippocampal activation in the active as compared to the sham tDCS condition could not be assessed. For this purpose, combined fMRI recording would have been necessary. However, in our view, establishing a behavioral stimulation effect had priority over neurophysiological effects, as a change in hippocampal activity without a change in memory performance would not have had the same relevance. Finally, the sleep conditions during the study procedure were far from the natural nightsleep setting. This setting might have altered the participants' sleep architecture and ultimately weakened our experimental effects. The upside of this limitation is that we have paved the way for further studies in the MRI environment.

Possible adjustments to the stimulation protocol used in this study include adapting not only the current density according to the SW response, but also the current flow direction. Moreover, instead of applying stimulation trains of two minutes, we might consider stimulation during the presence of SWs without interruption. The introduction of adaptive stimulation parameters could pave the way to closed-loop SW-stimulation. However, adaptive current density for instance does not necessarily enhance tDCS responsiveness or cortical excitability, as shown by Kidgell et al. [56].

\section{Conclusion}

The present study has shown that by applying tDCS only during SWS episodic memory consolidation was boosted in participants with a high baseline performance. Additionally, active tDCS elevated SWamps compared to sham. Despite the seemingly promising results that revealed a convergence of behavioral and electrophysiological dependent variables, the method applied had a limited effectiveness in our healthy sample, given average and lower memory performers did not benefit from stimulation. Moreover, this study adds evidence to previous reports showing a limited potential to augment the functionality of a healthy, highly specialized mechanism (i.e. 
system consolidation [6]). Therefore, further studies investigating whether applying the current experimental setup with patients with memory deficits would yield greater beneficial effects are warranted.

\section{Abbreviations}

$\mathrm{BOLD}=$ blood oxygen level dependent

CSF $=$ cerebral spinal fluid

DLPFC $=$ dorsolateral prefrontal cortex

$E E G=$ electroencephalogram

ESS $=$ Epworth sleepiness scale

FSS $=$ fatigue severity scale

$\mathrm{GM}=$ grey matter

fMRI = functional magnetic resonance imaging

MNI = Montreal Neurological Institute

$\mathrm{MRI}=$ magnetic resonance imaging

$\mathrm{N} 1=$ NREM sleep stage 1

$\mathrm{N} 2=$ NREM sleep stage 2

NREM $=$ non-rapid eye movement

$\mathrm{REM}=$ rapid eye movement

RLSSQ $=$ restless legs syndrome screening

questionnaire

SWamp = slow wave amplitude

SWS = slow wave sleep (NREM sleep stage 3)

tACS $=$ transcranial alternating current

stimulation

tDCS $=$ transcranial direct current stimulation

TES $=$ transcranial electric stimulation

$\mathrm{TST}=$ total sleep time

$\mathrm{W}=$ wake

$\mathrm{WM}=$ white matter

\section{Conflict of interest statement}

Financial Disclosure: none.

Non-financial Disclosure: Stephanie Winkelbeiner was funded by the Swiss National Science Foundation (\#320030_146789).

\section{References}

[1] Tulving E. Episodic memory: From mind to brain. Annu Rev Psychol 2002;53:1-25.
[2] Tulving E. Relations among Components and Processes of Memory. Behav Brain Sci 1984;7(2):257-63.

[3] Marr D. Simple Memory - Theory for Archicortex. Philos T Roy Soc B 1971;262(841):23-81.

[4] Rasch B, Born J. About sleep's role in memory. Physiol Rev 2013;93(2):681-766.

[5] Yonelinas AP, Ranganath C, Ekstrom AD, Wiltgen BJ. A contextual binding theory of episodic memory: systems consolidation reconsidered. Nat Rev Neurosci 2019;20(6):364-75.

[6] Born J, Wilhelm I. System consolidation of memory during sleep. Psychol Res 2012;76(2):192-203.

[7] Dang-Vu TT, Schabus M, Desseilles M, Sterpenich $\mathrm{V}$, Bonjean $\mathrm{M}$, Maquet $\mathrm{P}$. Functional neuroimaging insights into the physiology of human sleep. Sleep 2010;33(12):1589-603.

[8] Diekelmann S, Wilhelm I, Born J. The whats and whens of sleep-dependent memory consolidation. Sleep Med Rev 2009;13(5):309-21.

[9] Iber C. The AASM manual for the scoring of sleep and associated events: rules, terminology and technical specifications. Westchester, IL: American Academy of Sleep Medicine; 2007.

[10] Mander BA, Rao V, Lu B, Saletin JM, Lindquist JR, Ancoli-Israel $S$, et al. Prefrontal atrophy, disrupted NREM slow waves and impaired hippocampaldependent memory in aging. Nat Neurosci 2013;16(3):357-64.

[11] Schonauer M, Pawlizki A, Kock C, Gais $S$. Exploring the effect of sleep and reduced interference on different forms of declarative memory. Sleep 2014;37(12):19952007.

[12] Plihal W, Born J. Effects of early and late nocturnal sleep on declarative and procedural memory. J Cognitive Neurosci 1997;9(4):534-47. 
[13] Gais S, Lucas B, Born J. Sleep after learning aids memory recall. Learn Mem 2006;13(3):259-62.

[14] Marshall L, Helgadottir H, Molle M, Born J. Boosting slow oscillations during sleep potentiates memory. Nature 2006;444(7119):610-3.

[15] Marshall L, Molle M, Hallschmid M, Born J. Transcranial direct current stimulation during sleep improves declarative memory. J Neurosci 2004;24(44):9985-92.

[16] Reis J, Schambra HM, Cohen LG, Buch ER, Fritsch B, Zarahn E, et al. Noninvasive cortical stimulation enhances motor skill acquisition over multiple days through an effect on consolidation. Proc Natl Acad Sci U S A 2009;106(5):1590-5.

[17] Robison MK, McGuirk WP, Unsworth N. No Evidence for Enhancements to Visual Working Memory With Transcranial Direct Current Stimulation to Prefrontal or Posterior Parietal Cortices. Behav Neurosci 2017;131(4):277-88.

[18] Passmann S, Kulzow N, Ladenbauer J, Antonenko D, Grittner U, Tamm S, et al. Boosting Slow Oscillatory Activity Using tDCS during Early Nocturnal Slow Wave Sleep Does Not Improve Memory Consolidation in Healthy Older Adults. Brain Stimul 2016;9(5):730-9.

[19] Savic B, Muri R, Meier B. A single session of prefrontal cortex transcranial direct current stimulation does not modulate implicit task sequence learning and consolidation. Brain Stimulation 2017;10(3):567-75.

[20] Sahlem GL, Badran BW, Halford JJ, Williams NR, Korte JE, Leslie $\mathrm{K}$, et al. Oscillating Square Wave Transcranial Direct Current Stimulation (tDCS) Delivered During Slow Wave Sleep Does Not Improve Declarative Memory More Than Sham: A Randomized Sham Controlled Crossover Study. Brain Stimul 2015;8(3):528-34.
[21] Ladenbauer J, Ladenbauer J, Kulzow N, de Boor R, Avramova E, Grittner U, et al. Promoting Sleep Oscillations and Their Functional Coupling by Transcranial Stimulation Enhances Memory Consolidation in Mild Cognitive Impairment. J Neurosci 2017;37(30):7111-24.

[22] Lafon B, Henin S, Huang Y, Friedman $\mathrm{D}$, Melloni L, Thesen T, et al. Low frequency transcranial electrical stimulation does not entrain sleep rhythms measured by human intracranial recordings. Nat Commun 2017;8(1):1199.

[23] Habich A, Klöppel S, Abdulkadir A, Scheller E, Nissen C, Peter J. Anodal tDCS Enhances Verbal Episodic Memory in Initially Low Performers. Front Hum Neurosci 2017;11(542).

[24] Rosen DS, Erickson B, Kim YE, Mirman D, Hamilton RH, Kounios J. Anodal tDCS to Right Dorsolateral Prefrontal Cortex Facilitates Performance for Novice Jazz Improvisers but Hinders Experts. Front Hum Neurosci 2016;10:579.

[25] Turkeltaub PE, Benson J, Hamilton RH, Datta A, Bikson M, Coslett HB. Left lateralizing transcranial direct current stimulation improves reading efficiency. Brain Stimulation 2012;5(3):201-7.

[26] Ke Y, Wang N, Du J, Kong L, Liu S, Xu $M$, et al. The Effects of Transcranial Direct Current Stimulation (tDCS) on Working Memory Training in Healthy Young Adults. Front Hum Neurosci 2019;13:19.

[27] Karabanov A, Thielscher A, Siebner HR. Transcranial brain stimulation: closing the loop between brain and stimulation. Curr Opin Neurol 2016;29(4):397-404.

[28] Buzsaki G. The hippocamponeocortical dialogue. Cereb Cortex 1996;6(2):81-92.

[29] Walker MP. The role of slow wave sleep in memory processing. J Clin Sleep Med 2009;5(2 Suppl):S20-6.

[30] Gagnon G, Blanchet S, Grondin S, Schneider C. Paired-pulse transcranial magnetic stimulation over the dorsolateral 
prefrontal cortex interferes with episodic encoding and retrieval for both verbal and non-verbal materials. Brain Res 2010;1344:148-58.

[31] Spaniol J, Davidson PS, Kim AS, Han H, Moscovitch M, Grady CL. Event-related fMRI studies of episodic encoding and retrieval: meta-analyses using activation likelihood estimation. Neuropsychologia 2009;47(8-9):1765-79.

[32] Barham MP, Enticott PG, Conduit R, Lum JAG. Transcranial electrical stimulation during sleep enhances declarative (but not procedural) memory consolidation: Evidence from a metaanalysis. Neurosci Biobehav R 2016;63:6577.

[33] Genzel L, Kiefer T, Renner L, Wehrle R, Kluge $M$, Grozinger $M$, et al. Sex and modulatory menstrual cycle effects on sleep related memory consolidation. Psychoneuroendocrinology 2012;37(7):98798.

[34] Phillips PJ, Wechsler H, Huang J, Rauss PJ. The FERET database and evaluation procedure for face-recognition algorithms. Image Vision Comput 1998;16(5):295-306.

[35] Phillips PJ, Moon H, Rizvi SA, Rauss PJ. The FERET evaluation methodology for face-recognition algorithms. Ieee T Pattern Anal 2000;22(10):1090-104.

[36] Ruch S, Zust MA, Henke K. Subliminal messages exert long-term effects on decision-making. Neurosci Conscious 2016;2016(1):niwo13.

[37] Svoboda E, McKinnon MC, Levine B. The functional neuroanatomy of autobiographical memory: a meta-analysis. Neuropsychologia 2006;44(12):2189-208.

[38] Andrade KC, Spoormaker VI, Dresler M, Wehrle R, Holsboer F, Samann PG, et al. Sleep spindles and hippocampal functional connectivity in human NREM sleep. J Neurosci 2011;31(28):10331-9.

[39] Van Essen DC, Smith SM, Barch DM, Behrens TEJ, Yacoub E, Ugurbil K, et al. The
WU-Minn Human Connectome Project: An overview. Neuroimage 2013;80:62-79.

[40] Glasser MF, Sotiropoulos SN, Wilson JA, Coalson TS, Fischl B, Andersson JL, et al. The minimal preprocessing pipelines for the Human Connectome Project. Neuroimage 2013;80:105-24.

[41] Eickhoff SB, Stephan KE, Mohlberg H, Grefkes C, Fink GR, Amunts K, et al. A new SPM toolbox for combining probabilistic cytoarchitectonic maps and functional imaging data. Neuroimage 2005;25(4):132535.

[42] Nitsche MA, Paulus W. Sustained excitability elevations induced by transcranial DC motor cortex stimulation in humans. Neurology 2001;57(10):1899-901.

[43] Fregni F, Boggio PS, Nitsche $M$, Bermpohl F, Antal A, Feredoes E, et al. Anodal transcranial direct current stimulation of prefrontal cortex enhances working memory. Experimental Brain Research 2005;166(1):23-30.

[44] Hoy KE, Arnold SL, Emonson MRL, Daskalakis ZJ, Fitzgerald PB. An investigation into the effects of tDCS dose on cognitive performance over time in patients with schizophrenia. Schizophr Res 2014;155(1-3):96-100.

[45] Shin YI, Foerster A, Nitsche MA. Transcranial direct current stimulation (tDCS) - Application in neuropsychology. Neuropsychologia 2015;69:154-75.

[46] Jacobson L, Koslowsky M, Lavidor M. tDCS polarity effects in motor and cognitive domains: a meta-analytical review. Exp Brain Res 2012;216(1):1-10.

[47] Guleyupoglu B, Febles N, Minhas P, Hahn C, Bikson M. Reduced discomfort during high-definition transcutaneous stimulation using $6 \%$ benzocaine. Front Neuroeng 2014;7:28.

[48] Lau H, Tucker MA, Fishbein W. Daytime napping: Effects on human direct associative and relational memory. Neurobiol Learn Mem 2010;93(4):554-60. 
[49] Tucker MA, Fishbein W. Enhancement of declarative memory performance following a daytime nap is contingent on strength of initial task acquisition. Sleep 2008;31(2):197-203.

[50] Hayes AF. Introduction to Mediation, Moderation, and Conditional Process Analysis: A Regression-Based Approach. New York, NY: The Guilford Press; 2013.

[51] Bauer DJ, Curran PJ. Probing interactions in fixed and multilevel regression: Inferential and graphical techniques. Multivar Behav Res 2005;40(3):373-400.

[52] Czisch M, Wehrle R. EEG-fMRI: Sleep. Heidelberg: Springer; 2009.

[53] Reinhart RMG, Xiao WX, McClenahan LJ, Woodman GF. Electrical Stimulation of
Visual Cortex Can Immediately Improve Spatial Vision. Curr Biol 2016;26(14):186772.

[54] Diekelmann S, Born J. The memory function of sleep. Nat Rev Neurosci 2010;11(2):114-26.

[55] Bruckner S, Kammer T. Both anodal and cathodal transcranial direct current stimulation improves semantic processing. Neuroscience 2017;343:269-75.

[56] Kidgell DJ, Daly RM, Young K, Lum J, Tooley G, Jaberzadeh S, et al. Different Current Intensities of Anodal Transcranial Direct Current Stimulation Do Not Differentially Modulate Motor Cortex Plasticity. Neural Plasticity 2013. 


\section{Supplementary Material}

A

bilateral hippocampus mask

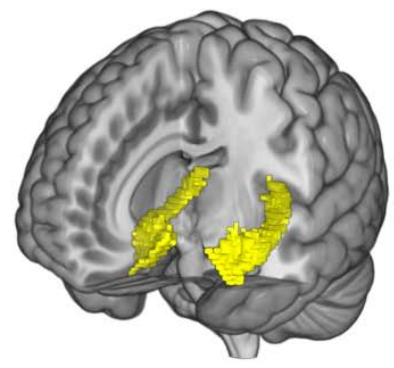

$\mathbf{R}$
B hippocampal functional connectivity (FWE-corrected $p<0.05)$

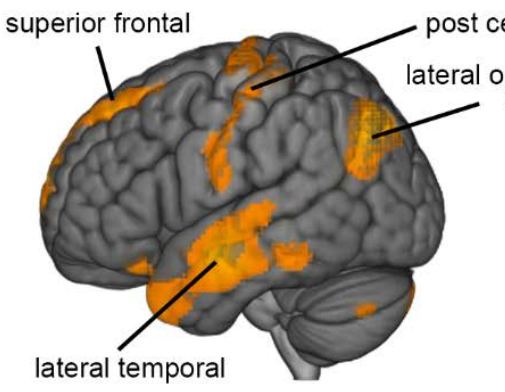

L

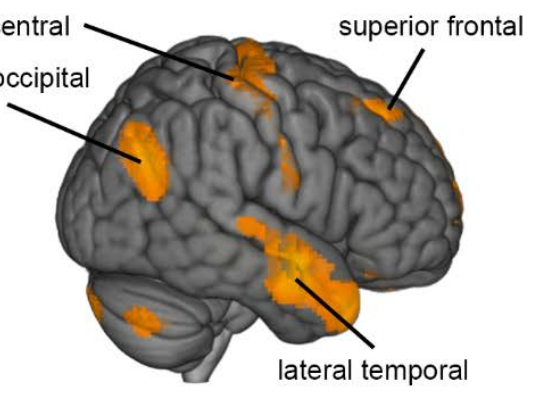

$\mathbf{R}$

Figure S1: (A) Illustration of the hippocampal mask that was used as the seed region. (B) Significantly connected regions located on the surface of the cortex.

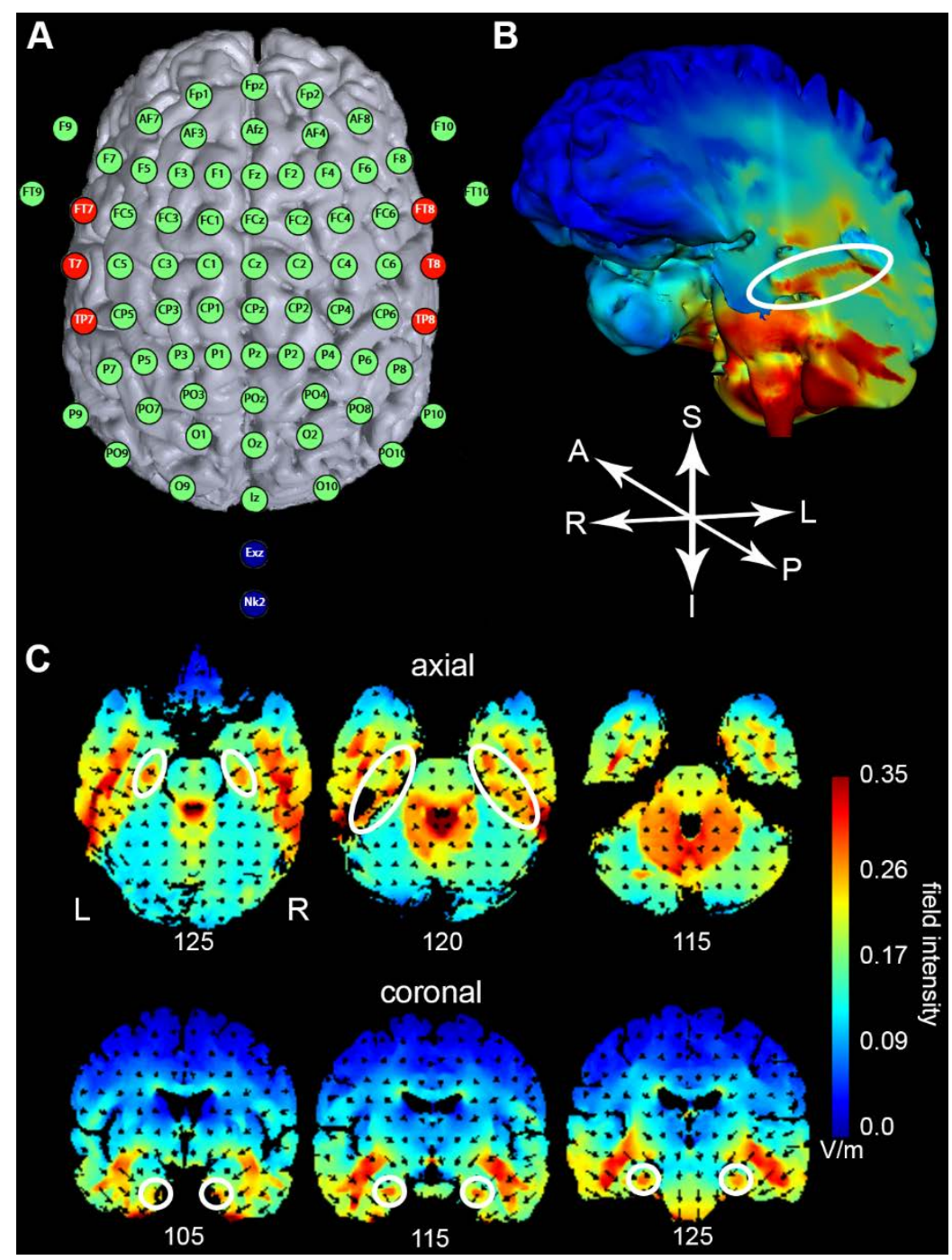

Figure S2: (A) Schematic tDCS montage integrated into the EEG 10-20 system. (B) Lateral cut along the hippocampus shows simulated increased electrical field intensity (white ellipse). (C) Axial and coronal slices illustrating increased simulated electrical field intensities in hippocampal areas (white ellipses and circles). $\mathrm{A}=$ anterior; $\mathrm{I}=$ inferior; $\mathrm{L}=$ left; $\mathrm{P}=$ posterior; $\mathrm{R}=$ right; $\mathrm{S}=$ superior. 
Table S1: SPM output list of significant clusters that are functionally connected with the seed mask (i.e. bilateral hippocampus).

\begin{tabular}{|c|c|c|c|c|c|c|}
\hline \multicolumn{2}{|c|}{ cluster-level } & \multicolumn{2}{|c|}{ peak-level } & \multirow{2}{*}{$\mathrm{x}$} & \multirow{2}{*}{$\mathrm{y}$} & \multirow{2}{*}{ z } \\
\hline PFWE-corr & $\mathrm{K}_{\mathrm{E}}$ & $\mathrm{p}_{\text {FWE-corr }}$ & $T$ & & & \\
\hline$<0.001$ & 325290 & $<0.001$ & 33.72 & -26 & -16 & -18 \\
\hline$<0.001$ & 1643 & $<0.001$ & 15.86 & -42 & -70 & 36 \\
\hline$<0.001$ & 1322 & $<0.001$ & 13.28 & 52 & -70 & 34 \\
\hline$<0.001$ & 376 & $<0.001$ & 12.10 & -14 & -88 & -40 \\
\hline$<0.001$ & 486 & $<0.001$ & 12.01 & 14 & -86 & -38 \\
\hline$<0.001$ & 881 & $<0.001$ & 11.41 & 22 & 32 & 44 \\
\hline$<0.001$ & 759 & $<0.001$ & 10.78 & -6 & -50 & -42 \\
\hline$<0.001$ & 346 & $<0.001$ & 10.53 & 42 & -70 & -36 \\
\hline$<0.001$ & 5085 & $<0.001$ & 9.95 & 36 & -26 & 56 \\
\hline$<0.001$ & 94 & $<0.001$ & 7.93 & 44 & -20 & 22 \\
\hline$<0.001$ & 99 & $<0.001$ & 7.65 & -46 & -70 & -38 \\
\hline$<0.001$ & 38 & $<0.001$ & 6.62 & 0 & -22 & -20 \\
\hline$<0.001$ & 46 & 0.001 & 6.26 & 0 & -34 & -24 \\
\hline
\end{tabular}

Table S2: Numbers and percentages of the participants who correctly identified the stimulation condition (Identified) or both conditions correctly (all correct).

\begin{tabular}{llllll}
\hline & & \multicolumn{2}{c}{ identified } & \multicolumn{2}{c}{ all correct } \\
\cline { 3 - 6 } & $\mathrm{N}$ & $\%$ & $\mathrm{~N}$ & $\%$ \\
\hline $\mathrm{N}=20$ & active & 10 & 50.0 & 7 & \multirow{2}{*}{36.8} \\
$\mathrm{~N}=19$ & sham & 10 & 52.6 & & \\
\hline
\end{tabular}

Note: stimulation condition guess of one participant was missing in the sham condition.

Table S3: Conditional effect of tDCS at values of the baseline memory performance (PERF1):

\begin{tabular}{rrrrrrr}
\hline PERF1 & Effect & SE & $\mathrm{t}$ & $\mathrm{p}$ & $\mathrm{LLCl}$ & $\mathrm{ULCl}$ \\
\hline 3.0000 & -4.0349 & 1.7199 & -2.3460 & 0.0246 & -7.5231 & -0.5468 \\
3.8500 & -3.6081 & 1.5905 & -2.2686 & 0.0294 & -6.8338 & -0.3824 \\
4.7000 & -3.1813 & 1.4640 & -2.1730 & 0.0364 & -6.1505 & -0.2121 \\
5.5500 & -2.7544 & 1.3413 & -2.0535 & 0.0473 & -5.4748 & -0.0340 \\
5.7074 & -2.6754 & 1.3191 & -2.0281 & 0.0500 & -5.3507 & 0.0000 \\
6.4000 & -2.3276 & 1.2236 & -1.9022 & 0.0652 & -4.8093 & 0.1541 \\
7.2500 & -1.9007 & 1.1124 & -1.7086 & 0.0961 & -4.1569 & 0.3554 \\
8.1000 & -1.4739 & 1.0099 & -1.4594 & 0.1531 & -3.5222 & 0.5744 \\
8.9500 & -1.0471 & 0.9190 & -1.1393 & 0.2621 & -2.9110 & 0.8168 \\
9.8000 & -0.6202 & 0.8434 & -0.7354 & 0.4669 & -2.3308 & 1.0904 \\
10.6500 & -0.1934 & 0.7876 & -0.2455 & 0.8074 & -1.7908 & 1.4040 \\
11.5000 & 0.2334 & 0.7560 & 0.3088 & 0.7593 & -1.2998 & 1.7667 \\
12.3500 & 0.6603 & 0.7516 & 0.8785 & 0.3855 & -0.8640 & 2.1845 \\
13.2000 & 1.0871 & 0.7748 & 1.4030 & 0.1692 & -0.4843 & 2.6586 \\
14.0500 & 1.5140 & 0.8234 & 1.8386 & 0.0742 & -0.1561 & 3.1840 \\
14.5009 & 1.7404 & 0.8581 & 2.0281 & 0.0500 & 0.0000 & 3.4808 \\
14.9000 & 1.9408 & 0.8933 & 2.1727 & 0.0365 & 0.1291 & 3.7524 \\
15.7500 & 2.3676 & 0.9798 & 2.4165 & 0.0209 & 0.3805 & 4.3547 \\
16.6000 & 2.7945 & 1.0790 & 2.5899 & 0.0138 & 0.6062 & 4.9828 \\
17.4500 & 3.2213 & 1.1877 & 2.7122 & 0.0102 & 0.8125 & 5.6301 \\
18.3000 & 3.6481 & 1.3035 & 2.7987 & 0.0082 & 1.0044 & 6.2919 \\
19.1500 & 4.0750 & 1.4248 & 2.8601 & 0.0070 & 1.1854 & 6.9646 \\
20.0000 & 4.5018 & 1.5501 & 2.9041 & 0.0063 & 1.3579 & 7.6457 \\
\hline
\end{tabular}

$\overline{\mathrm{SE}}=$ standard error; $\mathrm{LLCl}=$ lower limit of confidence interval; $\mathrm{ULCl}$ = upper limit of confidence interval. 